\title{
The NQR Observation of Spin-Peierls Transition in An Antiferromagnetic MX-Chain Complex \\ $\left[\mathrm{NiBr}(\operatorname{chxn})_{2}\right] \mathrm{Br}_{2}$
}

Shinya Takaishi, Yasuhiro Tobu, Hiroshi Kitagawa, Atsushi Goto, Tadashi Shimizu,

Takashi Okubo, Tadaoki Mitani, and Ryuichi Ikeda

Department of Chemistry, University of Tsukuba, Tsukuba 305-8571, Japan, National Institute for Materials Science, Tsukuba 305-0003, Japan, Japan Advanced Institute of Science and Technology, Tatsunokuchi, Ishikawa 923-1292, Japan

\section{$\underline{\text { Magnetic Susceptibility }}$}

Fig. S1 shows temperature dependence of magnetic susceptibilities $(\chi)$ observed in $\left[\mathrm{NiBr}(\operatorname{chxn})_{2}\right] \mathrm{Br}_{2} \cdot \chi$ data above $100 \mathrm{~K}$ were fitted by the recently proposed Eggert-Affleck-Takahashi (EAT) theory ${ }^{1}$ with $J=2000 \mathrm{~K}(H=$ $\left.2 J \Sigma S_{\mathrm{i}} S_{\mathrm{i}+1}\right)$. This theory corrected the Bonner-Fisher type susceptibility curve ${ }^{2}$ for the spin $1 / 2$ Heisenberg antiferromagnetic chain in the low-temperature range $\left(T<0.2 J / K_{\mathrm{B}}\right)$. The reported $g$-values determined by $\mathrm{ESR}^{3}$ $\left(g_{/ /}=2.02\right.$ and $\left.g_{\perp}=2.17\right)$ were used as the fitting parameters.

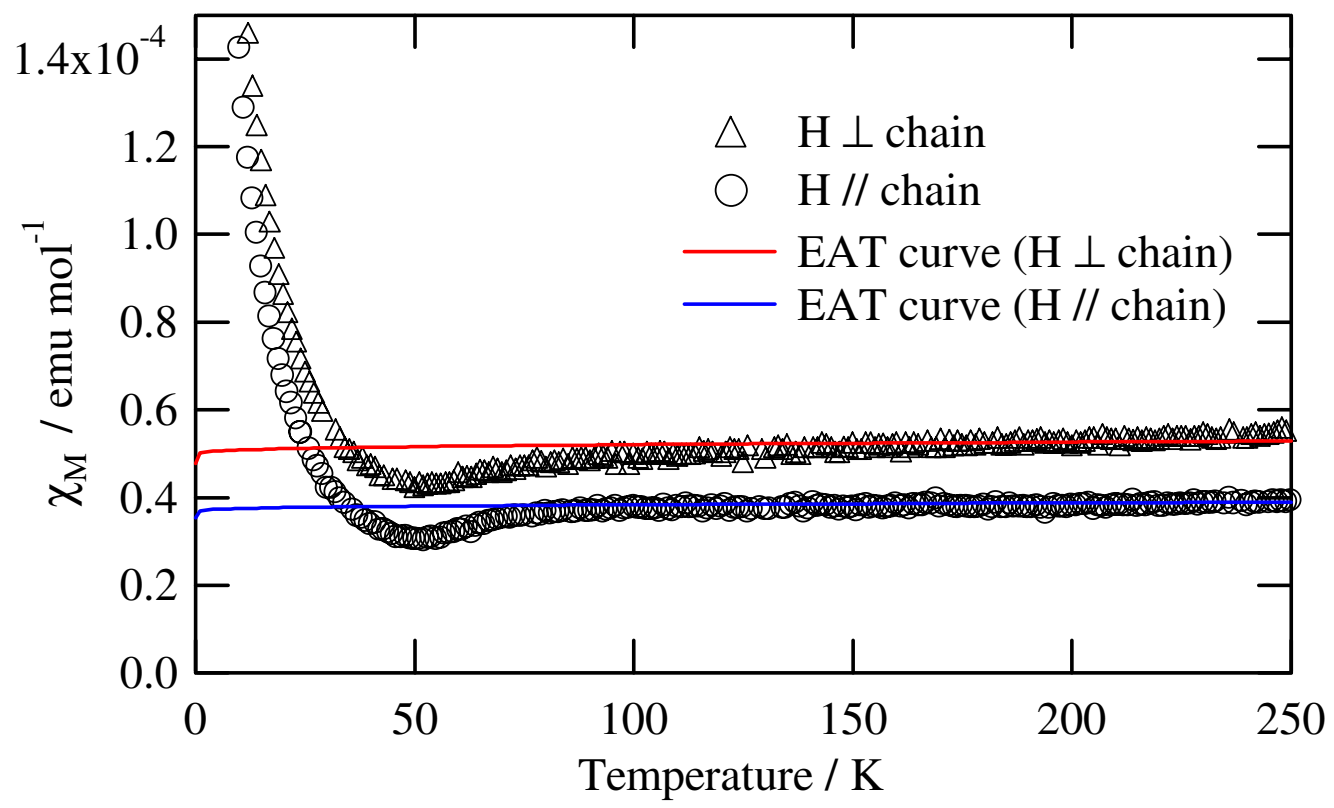

Figire S1 Magnetic susceptibilities observed in a single crystal of $\left[\mathrm{NiBr}(\operatorname{chxn})_{2}\right] \mathrm{Br}_{2}$ with a magnetic field $(1 \mathrm{~T})$ parallel and perpendicular to the 1-D chain. Red and blue lines show theoretical susceptibilities proposed by Eggerd-Affleck-Takahashi (EAT) with $J=2000 \mathrm{~K}$.

(1) Eggert, S; Affleck, I; and Takahashi, M Phys. Rev. Lett. 1994, 73, 332-335.

(2) Bonner J. C.; and Fisher, M. E. Phys. Rev. 1964, 135, A640-A658.

(3) Okamoto, H.; Toriumi, K.; Mitani, T.; Yamashita, M. Phys. Rev. B 1990, 42, 10381-10387. 